\title{
Improving Fast Atom Bombardment Mass Spectra: The Influence of Some Controllable Parameters on Spectral Quality
}

\author{
Johnny D. Reynolds and Kelsey D. Cook \\ Department of Chemistry, University of Tennessee, Knoxville, Tennessee, USA
}

The relative effects of adjustable fast atom bombardment (FAB) parameters (choice of matrix, primary atom flux, and primary atom energy) on the appearance of FAB spectra (including signal-to-noise, signal-to-background, and signal-to-matrix ratios) of several organic dyes have been investigated. Beam-induced chemical damage is minimized by lowering the primary atom flux, by raising the primary atom energy, and by selecting a matrix with radical scavenging properties (e.g., $m$-nitrobenzyl alcohol). The relative importance in minimizing this chemical damage is choice of matrix $>$ primary atom flux $>$ (nominal) primary atom energy, but optimization of the parameters involves a trade-off between sensitivity and damage. The effect of these parameters on thermal damage (fragmentation) is much less. It can be concluded from comparison of the dyes that the extent of beam damage does not depend simply on the standard reduction potential of the analyte. (J Am Soc Mass Spectrom 1990, 1, 149-157)

$\mathrm{T}$ The use of fast atom bombardment (FAB) [1] and liquid secondary ion [2] mass spectrometry (SIMS) for the characterization of nonvolatile and thermally labile compounds has developed rapidly over the past eight years. Optimally, the desorption ionization process generates molecular or quasimolecular ions as well as structurally significant fragments; however, many beam-induced reactions can occur that alter the structure of the analyte molecule. In addition to unimolecular fragmentation (a manifestation of beam-induced thermal "damage"), these reactions include adduct formation between the matrix and analyte [3], halogen replacement [4], reduction involving electrons $[5,6]$ and/or hydrogen atom(s) $[5,7]$, and metal exchange involving organometallics and the metallic FAB probe tip [8]. These latter reactions are all examples of beam-induced chemical damage, that is, damage resulting from intermolecular interactions with the products of thermal damage (fragments or electrons). In this context, the term "damage" is applied on a molecular scale, rather than with the macroscopic connotation of damage at or near the sample surface, as defined in dry SIMS studies.

Important parameters known to affect the extent of beam-induced damage and the abundance of molecular (or quasi-molecular) ions in FAB spectra include the primary beam flux [9-11] and energy [12, 13], and the matrix composition [3, 14-18]. Adventitious photons from the FAB gun may also be involved [9]. Most fundamental studies have used a "one-parameter-at-a-

Address reprint requests to Kelsey D. Cook, Department of Chemistry, University of Tennessee, Knoxville, TN 37996-1600. time" approach, usually assessing the effect of varying only a single parameter. While this has provided useful mechanistic insights, there is a significant danger of misinterpreting experimental data if the assumption of parameter independence breaks down. Furthermore, the relative importance of the various parameters has not been established.

Despite their importance in determining the appearance of a FAB mass spectrum, primary beam flux and energy are seldom reported accurately; often, information about either or both is omitted entirely. In reference to the flux, this is due at least in part to the difficulty of measurement. In a saddle field gun, only the emission current (i.e., the total current flowing between the anode and the cathode, measured as the total drain on the high-voltage power supply) is easily measured. Alexander and Hogg [19] established that a saddle field xenon atom gun operating at $7 \mathrm{keV}$ and $1 \mathrm{~mA}$ emission current provides a total primary beam current equivalent to only 10-15 $\mu \mathrm{A}$. Only about half of this current is due to neutral atoms; the remainder arises from ions that escape from the gun. The energy of the neutral species cannot be readily determined, but the majority (70\%) of the ions are singly charged and acquire energies corresponding to just $0.70-0.85$ of the anode potential $[19,20]$. When directed at a sample floating at a potential equal to or greater than the potential of the saddle field anode (as is the case for the experiments described in this paper), these primary ions should not affect the sputtering process because of deceleration. However, Alexander and Hogg [19] and Ligon and Dorn [20] showed that multiply charged ions formed in the discharge can acquire up to 5 times 
the energy corresponding to the anode potential. Although the role of these high-energy ions (comprising $15 \%$ of the total primary beam) in the sampling process has not been reported, it may be significant; ions partially or totally neutralized following acceleration [20] can promote high-energy processes distinct from those expected at the nominal primary atom energy $[12,13]$. Because of these uncertainties, part of this study involves a modest effort to assess semiquantitatively the operational characteristics of our xenon atom gun. More important, this study seeks to compare the effects of varying matrix and primary atom flux and energy on the appearance of FAB spectra for a series of organic dyes, with a principal aim of providing practical guidance to $F A B$ users.

Bombardment-induced ionization of these dyes has been widely reported $[5,9,21-28]$. It is generally accompanied by the addition of one or more hydrogen atoms to the molecular ion, which gives rise to ions one or two mass units heavier than the molecular or quasi-molecular ion, with intensities larger than would be predicted on the basis of natural isotopic abundances. This excess intensity results from beaminduced chemical damage, as defined above. Signal-tonoise and signal-to-background ratios (important, separate parameters often overlooked in damage studies) will also be assessed under various experimental conditions.

To supplement these studies, electrohydrodynamic (EH) mass spectrometry $[17,29]$ will be used to determine the extent of any solvent-induced reduction in the absence of bombardment. Electrohydrodynamic mass spectrometric sampling occurs via field evaporation, which imparts little excess energy to the system and thus avoids fragmentation. When reduction reactions have occurred in EHMS, they have been shown to reflect solution chemistry rather than sampling artifacts [17].

\section{Experimental}

\section{Fast Atom Bombardment Mass Spectra}

Fast atom bombardment spectra were obtained with a VG ZAB-EQ mass spectrometer operating at an accelerating potential of $8 \mathrm{kV}$ with a mass resolution of $1000-2000$ ( $10 \%$ valley definition). Research grade xenon (MG Industries) was used with an Ion Tech atom gun, operating with $1.0 \mathrm{~mA}$ emission current and 8.0 $\mathrm{keV}$ energy, except as noted.

The standard VG probe was modified to avoid loss of the tip in the ion source or inlet system as the probe was being inserted or removed. The new probe tip consists of a 3-mm-diameter cylindrical brass rod that is $10 \mathrm{~mm}$ in length. The tip is held with a set screw in a stainless steel cylinder that is attached to the insulator of the standard FAB probe. No noticeable difference was observed when spectra from the new and standard probe tips were compared.

Fast atom bombardment data were collected either by scanning the accelerating and ESA potentials (V- scans) or in the normal magnet scan mode. When precise intensity data were required for comparison with calculated isotopic distributions, V-scans were performed over a narrow mass range $(\sim 30 \mathrm{u})$, and data were collected by summing 16 or 25 scans (multichannel acquisition, MCA) over a period of 5-10 min. $V$-scans were used because they are more reproducible than magnet scans for MCA data acquisition. The results reported represent the average of at least three MCA spectra, each with a fresh sample (48 or 75 total scans). When a wider mass range was scanned, spectra from at least ten normal magnet scans were averaged. In all cases, the reported uncertainties represent the standard deviation of the mean $\left(s / n^{1 / 2}\right)$ of $n$ spectra. Estimated uncertainties in derived values (e.g., the relative enhancement factor) were determined by standard propagation-of-errors analysis [30]. In some cases, error bars were smaller than the symbols used to plot individual points.

Fast atom bombardment spectra of the dyes were obtained from a thin film $(5-20 \mu \mathrm{L})$ of standard solutions applied to the brass tip. Concentrations of these solutions varied from 1 to $30 \mathrm{mM}$ depending on dye solubility. However, a constant concentration was used for each dye throughout this study, regardless of matrix. Solutions were not degassed before analysis, but spectra were obtained only after emission had stabilized (typically 3-4 min for glycerol and 1-2 min for thioglycerol and $m$-nitrobenzyl alcohol).

\section{Electrohydrodynamic Mass Spectra}

Electrohydrodynamic spectra were obtained with an AEI MS-902 mass spectrometer equipped with a VG Update electronics console operating at an accelerating (emitter) potential of 6 or $8 \mathrm{kV}$, with slits fully open (resolution $\approx 400,10 \%$ valley definition) for maximum sensitivity. The design and operation of the source have been described elsewhere $[29,31]$. The emitter was a $200-\mu \mathrm{m}$-i.d. platinum capillary (Hamilton). Its potential was matched to the ESA bandpass by maximizing the signal intensity of the ion at $\mathrm{m} / \mathrm{z} 207$ $\left(\left[\mathrm{Na}+\mathrm{G}_{2}\right]^{+}\right.$, where $\mathrm{G}$ denotes glycerol). The EH extractor potential was between -1.0 and $-2.0 \mathrm{kV}$, and the collector was at ground potential.

Electrohydrodynamic spectra were collected using 10-s normal magnet scans. Data reported here represent the average of at least three scans, and uncertainties represent the standard deviation of the mean $\left(s / n^{1 / 2}\right)$ of $n$ spectra. Solutions were degassed before analysis for at least $8 \mathrm{~h}$ at low heat $\left(50^{\circ} \mathrm{C}\right)$ under vacuum $\left(10^{-3}\right.$ torr). In the EH experiments, dye concentrations were $0.05 \mathrm{~mol} \%(\approx 5 \mathrm{mM})$, and $\mathrm{NaCl}$ was used as supporting electrolyte to maintain the total ionic strength at $5 \mathrm{~mol} \%$ (glycerol $=100 \%$ ).

\section{Reagents}

Information concerning the dyes is summarized in Table 1. Glycerol was obtained from Sigma (Sigma grade). Thioglycerol (95\%), m-nitrobenzyl alcohol 
Table 1. Useful dye information

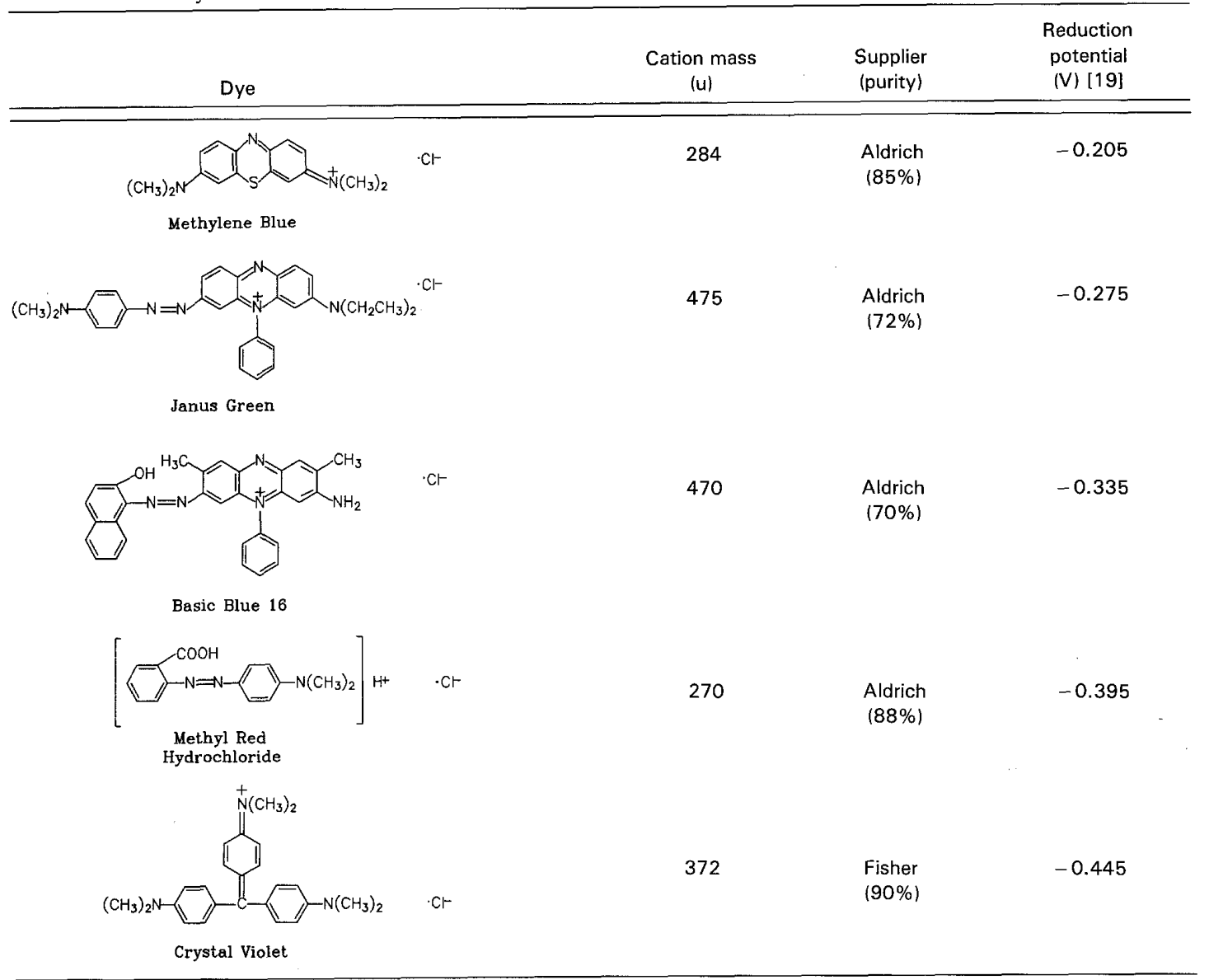

(98\%), CsI (reagent grade), and $\mathrm{NaCl}$ (reagent grade) were obtained from Aldrich. All reagents were used as received without further purification.

\section{Preliminary Experiments}

To characterize the FAB gun used in these studies, the intensity of a standard $\left(\mathrm{Cs}^{+} ; \mathrm{m} / \mathrm{z} 133\right.$ from $\left.\mathrm{CsI}\right)$ was monitored as a function of the emission current and primary atom energy, as suggested by Todd [11]. A dry CsI target was used for these studies to avoid the complications (e.g., changes in geometry and concentration) associated with solutions. Dry CsI offered the additional advantage that by direct observation of sample luminescence during bombardment (through the viewport in the $Z A B$ source housing), it was easily possible to confirm that the target was fully illuminated under all gun operating conditions, as expected for our FAB gun. While this does not eliminate changes in flux resulting from changes in focusing at different primary beam energies, it does reduce these effects and those arising from beam wander.

Intensity of $\mathrm{Cs}^{+}$was found to vary slightly each time the gun was turned on, even under conditions of constant emission current, gas pressure, and primary atom energy. The relative standard deviation (RSD) varied from $\pm 1 \%$ to $\pm 4 \%$, with the best precision occurring under conditions of high emission current and high primary energy $(1 \mathrm{~mA}$ and $8 \mathrm{keV})$. Plots of absolute $\mathrm{Cs}^{+}$ion intensity versus emission current $(0.2-1.0$ $\mathrm{mA})$ are linear and have increasing slopes (0.8 at 2 $\mathrm{keV}, 1.2$ at $5 \mathrm{keV}$, and 1.6 at $8 \mathrm{keV}$ ) for a range of primary beam energies. Correlation coefficients of the plots are $0.996,0.998$, and 0.999 for the primary beam energies of 2, 5, and $8 \mathrm{keV}$, respectively. Todd [11] showed that secondary ion intensity from a sample of glycerol bombarded by an argon ion source varies linearly with primary ion current density over the range $0.08-2 \mu \mathrm{A} / \mathrm{cm}^{2}$. Assuming similar proportionality between primary atom flux and secondary ion intensity for the system used here, it can be concluded that the primary atom flux should be proportional to the emission current. Furthermore, the atom gun should be tunable to a reproducible flux at a given energy by monitoring the intensity of the standard $\left(\mathrm{Cs}^{+}\right)$.

The ability to accurately determine isotopic contributions in FAB was assessed by examination of the $\mathrm{K}_{2} \mathrm{I}^{+}$cluster $(m / z 205,207,209)$ from $\mathrm{KI}$ at various 


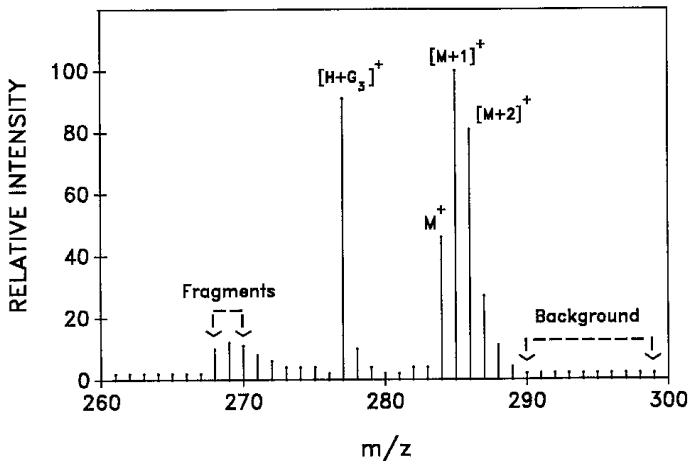

Figure 1. Mass spectrum of methylene blue in glycerol under standard FAB conditions ( $8 \mathrm{keV}, 1.0 \mathrm{~mA}$ emission current; G denotes glycerol).

primary atom fluxes and energies. Agreement with known isotopic distributions was found to be well within limits acceptable for detecting beam-induced reduction. The worst case occurred at $0.2 \mathrm{~mA}$ and 2.0 $\mathrm{keV}$, where the measured intensities were in the ratio 100:14.8:1.1 compared with natural isotopic abundances of 100:14.4:0.5 for the ions at $m / z 205,207$, and 209 , respectively.

Table 2. Intensities in the molecular ion region for various dyes sampled from glycerol by FAB/MS $(8 \mathrm{keV}$, $1.0 \mathrm{~mA}$ emission current) and by EHMS

\begin{tabular}{|c|c|c|c|}
\hline Dye & $\mathrm{EH}$ & FAB & Calc. \\
\hline \multicolumn{4}{|c|}{ Methylene blue } \\
\hline $\mathrm{M}^{+}$ & 100 & 100 & 100 \\
\hline$[\mathrm{M}+1]^{+}$ & $22 \pm 3$ & $212 \pm 16$ & 20 \\
\hline$[\mathrm{M}+2]^{+}$ & $9 \pm 3$ & $175 \pm 11$ & 6 \\
\hline \multicolumn{4}{|c|}{ Janus green } \\
\hline $\mathrm{M}^{+}$ & 100 & 100 & 100 \\
\hline$[\mathrm{M}+1]^{+}$ & $40 \pm 3$ & $249 \pm 18$ & 37 \\
\hline$[\mathrm{M}+2]^{+}$ & $9 \pm 3$ & $556 \pm 32$ & 6 \\
\hline \multicolumn{4}{|c|}{ Basic blue 16} \\
\hline $\mathbf{M}^{+}$ & 100 & 100 & 100 \\
\hline$[M+1]^{+}$ & $37 \pm 3$ & $168 \pm 14$ & 36 \\
\hline$[M+2]^{+}$ & $7 \pm 2$ & $227 \pm 20$ & 6 \\
\hline \multicolumn{4}{|c|}{ Methyl red hydrochloride ${ }^{a}$} \\
\hline $\mathrm{M}^{+}$ & 100 & 100 & 100 \\
\hline$[M+1]^{+}$ & $19 \pm 3$ & $164 \pm 10$ & 18 \\
\hline$[M+2]^{+}$ & $4 \pm 2$ & $253 \pm 15$ & 2 \\
\hline \multicolumn{4}{|c|}{ Crystal violet } \\
\hline $\mathrm{M}^{+}$ & 100 & 100 & 100 \\
\hline$[\mathrm{M}+1]^{+}$ & $33 \pm 4$ & $43 \pm 4$ & 30 \\
\hline$[\mathrm{M}+2]^{+}$ & $7 \pm 3$ & $37 \pm 4$ & 4 \\
\hline
\end{tabular}

a $\mathrm{M}^{+}$for methyl red hydrochloride corresponds to the protonated quasi-molecular ion.

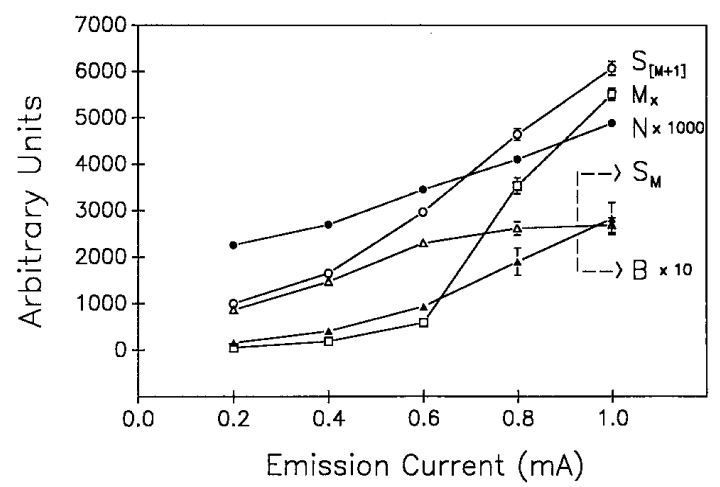

Figure 2. Signal $(S)$, noise $(N)$, background $(B)$, and matrix $\left(M_{x}\right)$ peak intensities versus $F A B$ gun emission current for methylene blue sampled from glycerol with $8 \mathrm{-keV}$ xenon atoms ( $B$ measured at $m / z=290 ; M_{x}$ measured at $m / z=277$, in all figures).

\section{Results and Discussion}

All but one of the dyes used contain quaternary nitrogens and yield molecular cations $\left([\mathrm{M}]^{+}\right)$under FAB conditions; methyl red hydrochloride is exceptional in that a quasi-molecular ion $\left([\mathrm{M}+\mathrm{H}]^{+}\right)$is most abundant in the spectrum (Table 1). Under standard FAB operating conditions ( $8 \mathrm{keV}$ and $1.0 \mathrm{~mA}$ ), each dye spectrum shows intensities higher than expected 1 and $2 \mathrm{u}$ above the molecular or quasi-molecular ion (Figure 1 and Table 2).

Electrohydrodynamic spectra confirm that all dyes are reasonably pure and are stable in glycerol (Table 2). Thus, impurities or spontaneous reduction by the glycerol solvent are not responsible for excess FAB intensities at $[\mathrm{M}+1]^{+}$and $[\mathrm{M}+2]^{+}$; beam-induced reduction is clearly evident. In probing this (and related thermal) damage, data for methylene blue are representative of the behavior of all dyes tested; therefore only data for this dye are presented in detail in the discussion that follows.

\section{Effect of Primary Flux}

As observed for the standard $\left(\mathrm{Cs}^{+}\right)$described earlier, the signal due to analyte would generally be expected to increase linearly with increasing flux. The resulting increase in signal-to-noise ratio $(S / N$, where $N$ is the standard deviation of a given analyte signal) accounts for the fact that most FAB experiments are performed at relatively high primary fluxes. The behavior of these dyes, however, is much more complex. For example, the molecular ion signal $\left(S_{\mathrm{M}^{+}} ; m / z 284\right)$ for methylene blue levels off at higher fluxes, while $S_{[\mathrm{M}+1]^{+}}(\mathrm{m} / \mathrm{z}$ 285 ) increases more rapidly than expected (Figure 2). This behavior results from the beam-induced reduction, which offsets the expected increase in $S_{\mathrm{M}^{+}}$while inflating $S_{[\mathrm{M}+1]}+$ at high fluxes. It is significant that this reduction increases with flux rather than comprising a fixed fraction of the secondary ion yield independent 


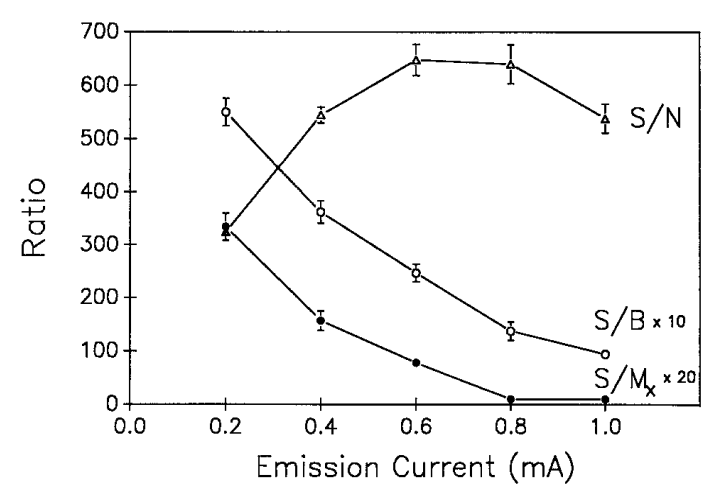

Figure 3. Ratios $S / N, S / B$, and $S / M_{x}$ versus $F A B$ gun emission current for methylene blue sampled from glycerol with $8-\mathrm{keV}$ xenon atoms.

of flux. Also, a slight increase in noise (standard deviation of intensity at $\mathrm{m} / \mathrm{z} 284$ ) is observed with increasing flux. More important are the increase in chemical noise or background $(B)$ (peak at every mass; assessed at $\mathrm{m} / \mathrm{z} 290$ ) and a more substantial increase in the intensity of major matrix ions $\left(\mathrm{M}_{x}\right.$, assessed at $m / z$ 277; $\left[\mathrm{G}_{3}+\mathrm{H}\right]^{+}$) that accompany increasing primary atom flux (Figure 2). These increases are not simple linear effects. They may arise from a higher proportion of multiply-charged, high-energy ions occurring at higher flux or from some sort of a cooperative effect.

Analytical sensitivity can be greatly affected by this complicated beam-induced behavior. The $S / N$ ratio for $\mathrm{m} / \mathrm{z} 284$ actually passes through a maximum as the primary atom flux is increased (Figure 3). Also, the decrease in signal-to-background ( $S / B ; \mathrm{m} / \mathrm{z} 284: \mathrm{m} / \mathrm{z} 290)$ and signal-to-matrix $\left(S / M_{x} ; m / z\right.$ 284:m/z 277) ratios with increasing primary flux (Figure 3) should cause the limits of detection (LOD) to increase with increasing primary flux if detection is background-limited (rather than noise-limited), which is usually the case in FAB.

In addition to standard analytical figures of merit, it is important to consider the dependence of beaminduced damage on the primary atom flux. The extent of reduction provides a convenient measure of chemical damage and can be quantitated using a relative enhancement factor, $E_{\mathrm{q}}$ :

$$
E_{\mathrm{q}}=\left(i_{\mathrm{m}, \mathrm{q}}-i_{\mathrm{c}, \mathrm{q}}\right) / i_{\mathrm{c}, \mathrm{q}}
$$

where $i_{\mathrm{m}, \mathrm{q}}$ is the measured peak intensity for ion $\mathrm{q}$ $\left([\mathrm{M}+1]^{+}\right.$or $\left.[\mathrm{M}+2]^{+}\right)$and $i_{\mathrm{c}, q}$ is the corresponding expected peak intensity for ion $\mathrm{q}$ based on natural isotopic abundances for $\mathrm{M}^{+}$. As the atom flux increases, so do $E_{[\mathrm{M}+1]^{+}}$and $E_{[\mathrm{M}+2]^{+}}$(Figure 4). Consistent with results of Todd and Groenewold [32], this suggests an increase in the abundance of thermal damage products (ions, radicals, and electrons; "reagents" in the reduction reaction). However, reduction of the primary flux during bombardment of a sample is followed instantly by a reduction in $E_{\mathrm{q}}$. Damage products must be removed from the sample surface quickly by one or more mechanisms (e.g., sputtering, evaporation, reaction, diffusion, or field emission). Brown and Busch [9] and Castro et al. [10] observed similar effects.

Clearly, reductive damage complicates the interpretation of FAB spectra. Arguably, the aromatic dyes used in this study may be particularly susceptible to this chemical beam-induced damage complication. By comparison, fragmentation represents a more general manifestation of beam-induced damage, one arising from thermal effects. For methylene blue, loss of $\mathrm{CH}_{4}$ generates the most abundant fragment in FAB [25], SIMS [23], and MS/MS [25]. The relative abundance of this fragmentation $(F)$ can be used as a measure of thermal damage:

$$
F=i_{\mathrm{f}} / i_{\mathrm{p}}
$$

where $i_{\mathrm{p}}$ is the sum of intensities for the precursor ions $(\mathrm{M}, \mathrm{M}+1$, and $\mathrm{M}+2)$, and $i_{\mathrm{f}}$ is the sum of intensities for ions arising from $\mathrm{CH}_{4}$ loss from the precursor ions. $F$ increases only slightly with increasing primary flux (Figure 4), as observed by Winger et al. [13]. Therefore, fragmentation is evidently less sensitive than reduction to primary atom flux. This is reasonable, because fragmentation is largely unimolecular whereas chemical damage is not.

One final effect of flux variation deserves mention. Because of reduced sputtering, sample persistence is greater at lower fluxes. For example, the lifetime of measurable signal from pure glycerol increases roughly three-fold (from $\sim 15 \mathrm{~min}$ to $\sim 45 \mathrm{~min}$ ) as the primary atom flux is lowered from $1.0 \mathrm{~mA}$ to $0.2 \mathrm{~mA}$ under conditions of constant primary beam energy $(8$ $\mathrm{keV}$ ). Although the lifetime of measurable analyte signals will vary from sample to sample (due to changes in surface activity, volatility, etc.), it will invariably be inversely proportional to the primary atom flux. This can be important when limited amounts of the analyte are available and long duration signals are needed, as when MS/MS experiments are being attempted.

\section{Effect of Primary Energy}

As observed elsewhere $[12,13]$, absolute ion signal increases as the primary atom beam energy increases, probably owing to increased depth of penetration [33, 34] (Figure 5). $S_{\mathrm{M}^{+}}$increases somewhat more rapidly than $S_{[\mathrm{M}+1]^{+}}$; the extent of reduction is evidently less at higher primary beam energies (Figure 6). Relative fragmentation $(F)$ is also slightly reduced at higher energies (Figure 6). These reductions in beam-induced damage may be explicable on the basis of the relationship between the primary atom energy and the sampling volume [13], although this is not a well-resolved issue [35]. Alternatively, this may arise from changes in beam focusing (and therefore flux) with changing primary beam energy. The accompanying increase in noise $(N$; Figure 5) may result from more "violent" and erratic sample sputtering at high energy. 


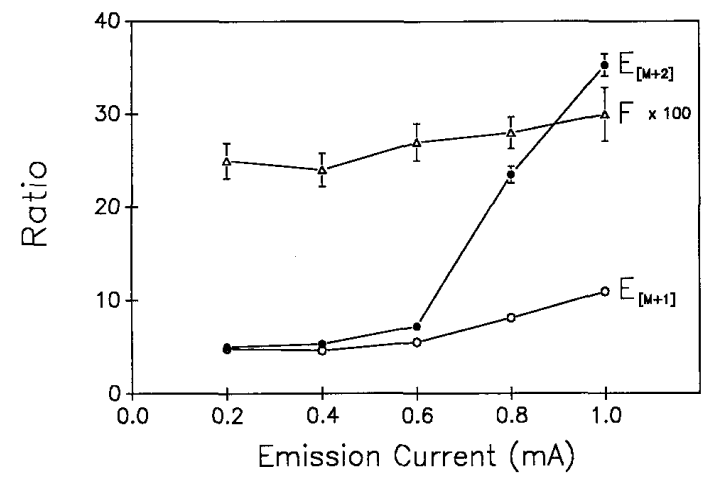

Figure 4. Extent of reduction ( $E_{\mathrm{q}}$, for the subscripted ion $\left.\mathrm{q}\right)$ and fragmentation $(F)$ versus FAB gun emission current for methylene blue sampled from glycerol with $8-\mathrm{keV}$ xenon atoms.

Background $(B)$ and matrix peaks $\left(M_{x}\right)$ also increase with increasing primary energy (Figure 5 ) but to a lesser extent than observed when the primary atom flux is increased. Overall, $S / B$ and $S / M_{x}$ ratios improve at lower energies, while $S / N$ deteriorates (Figure 7 ). The reduction in $S / N$ is probably due to signal attenuation by increased chemical and thermal damage at lower energies. If $S / B$ or $S / M_{x}$ (rather than $S / N$ ) is sensitivitylimiting, a sample-dependent trade-off between limit of detection and extent of damage will result.

As in the flux studies, the extent of reduction adjusts quickly to reflect changes in primary beam energy; reductive species do not appear to accumulate in solution. Also, as a result of decreased sputter rate, sample persistence is longer at lower energy despite enhanced damage. The lifetime of a signal from pure glycerol nearly doubles (from $\sim 15 \mathrm{~min}$ to $\sim 25 \mathrm{~min}$ ) as the energy is reduced from $8 \mathrm{keV}$ to $2 \mathrm{keV}$ under conditions of constant emission current $(1 \mathrm{~mA})$. As reasoned above, persistence of analyte signals should also be inversely proportional to the primary atom energy.

\section{Parameter Interaction}

It is evident from Figure 8 that there are complex interactions between the effects of primary atom flux and energy. No simple combination of these variables (e.g., power $=$ flux $\times$ energy) can readily predict the changes in reduction, fragmentation, $S / N, S / B$, or $S / M_{x}$ that accompany significant changes in primary atom flux or energy. Thus, it does not appear possible to prescribe a standard approach to optimization. The following generalizations do, however, appear justified:

1. Chemical damage (reduction) is reduced by lowering the primary atom flux and raising the primary atom energy.

2. Thermal damage (fragmentation) is affected to a much lesser extent but also appears to be mitigated at low flux and high energy.

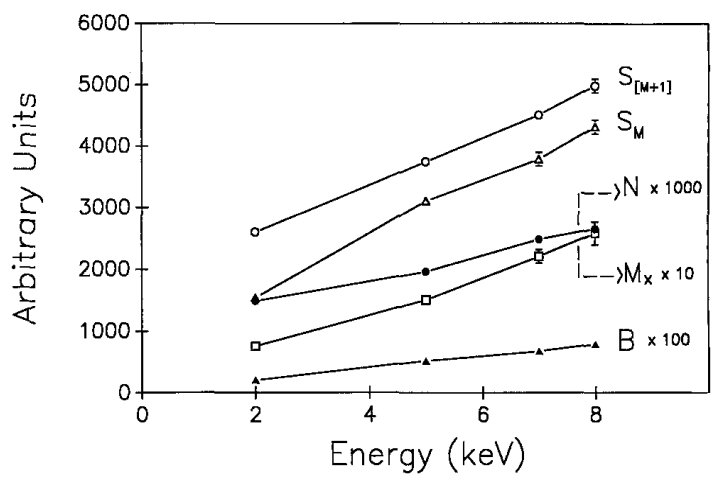

Figure 5. Signal $(S)$, noise $(N)$, background $(B)$, and matrix $M_{x}$ peak intensities versus primary beam energy for methylene blue sampled from glycerol at a constant emission current of $0.2 \mathrm{~mA}$.

3. When damage is extensive, the effect of varying primary atom flux is proportionately larger than that of varying primary atom energy (e.g., halving the flux mitigates chemical damage more effectively than does doubling the energy).

4. Beam-induced damage mitigation can be accompanied by deterioration of $S / N$, resulting in a trade-off in cases with noise-limited LOD.

5. On the other hand, in the more common (in FAB) case where $S / B$ determines the LOD, reduced flux will both reduce beam-induced damage and improve $S / B$ (therefore LOD); energy optimization will involve a trade-off since damage and $S / B$ both increase with decreasing energy. The effect will be most important for chemically labile samples (i.e., those which, like the dye samples in this study, are susceptible to chemical damage).

The two lowest curves of Figure 8 approach a limiting minimum value of $E_{\mathrm{q}}$ (near 5). If the other curves also approach this limiting value, they must do so at lower fluxes. This suggests that the current at which this limiting value is reached is shifted to lower val-

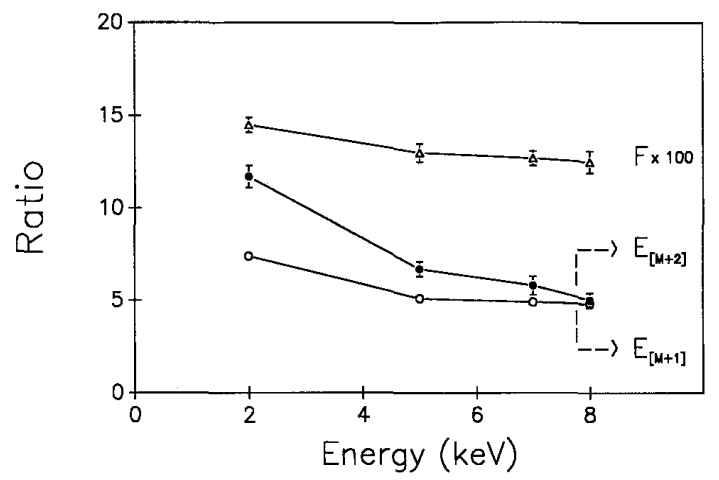

Figure 6. Extent of reduction ( $E_{\mathrm{q}}$, for the subscripted ion $\left.\mathrm{q}\right)$ and fragmentation $(F)$ versus primary beam energy for methylene blue sampled from glycerol at a constant emission current of 0.2 $\mathrm{mA}$. 


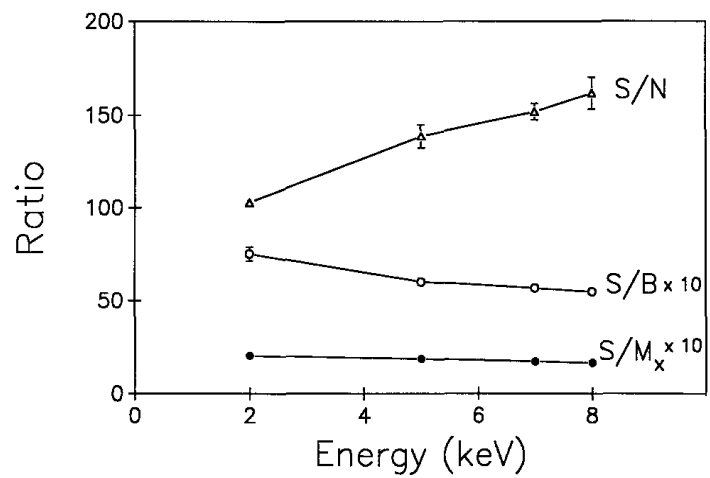

Figure 7. Ratios $S / N, S / B$, and $S / M_{x}$ versus primary beam energy for methylene blue sampled from glycerol at a constant emission current of $0.2 \mathrm{~mA}$.

primary energy is decreased. Below this value, sensitivity should suffer as $S / N$ eventually becomes limiting.

\section{Effect of Matrix Changes}

An important aspect of FAB is the mitigation of beaminduced damage by the "healing" action of the solvent (matrix). However, the solvent $[26,27]$ can also serve as a source of electrons and radicals (including hydrogen atoms) for chemical reduction of analytes like those considered here. The balance between healing and reductive properties is known to vary among common FAB matrices; $m$-nitrobenzyl alcohol [13] and thioglycerol [15] are noted for their radical-scavenging characteristics. Indeed, reduction is mitigated (Figure 9) when the dyes are sampled from thioglycerol rather than glycerol. When $m$-nitrobenzyl alcohol is used as the matrix, reduction is almost totally eliminated, and ion intensities approach those predicted on the basis of natural isotopic distributions 'Figure 9). From these data, reducing strength of these matrices can be ranked glycerol $>$ thioglycerol $>m$-nitrobenzyl alcohol. This is consistent with work by Miller et al. [14],

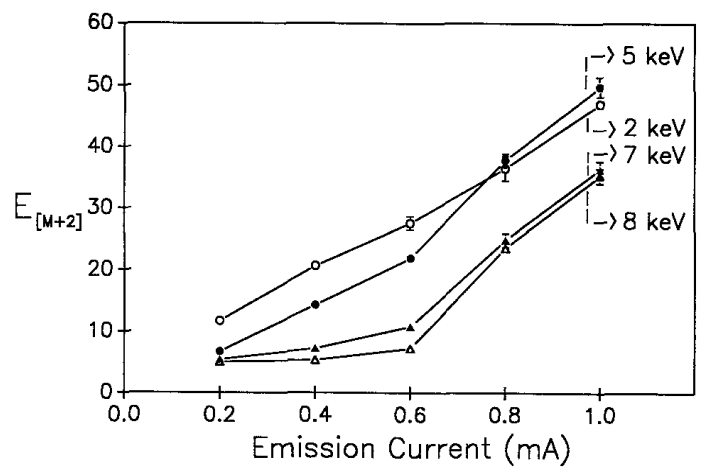

Figure 8. Extent of reduction $\left(E_{[M+2\}^{+}}\right)$versus $F A B$ gun emission current for methylene blue in glycerol at various primary beam energies.

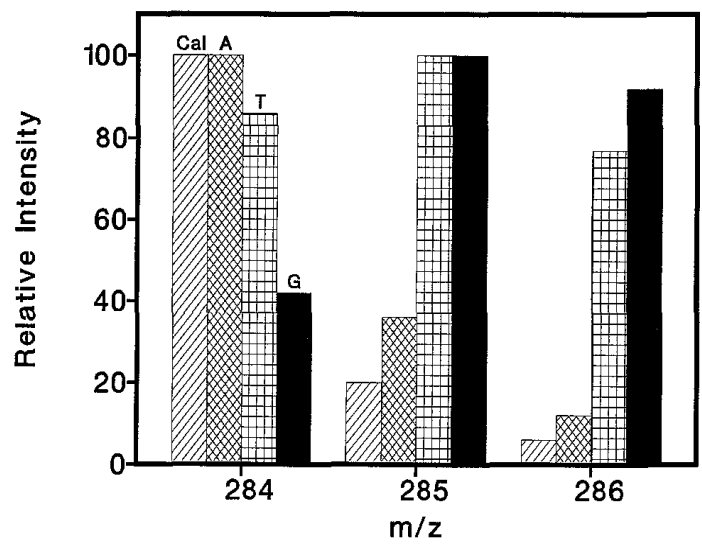

Figure 9. Fast atom bombardment intensities in the molecular ion region for methylene blue sampled under standard FAB conditions (1.0 mA and $8 \mathrm{keV}$ ) from glycerol $(\mathrm{G})$, thioglycerol $(\mathrm{T})$, and $m$-nitrobenyzl alcohol (A). Also included is the natural isotopic distribution (Cal).

who report that $m$-nitrobenzyl alcohol mitigates chemical damage by acting as an "electron sink."

Perhaps surprisingly, even thermal damage (as evident from fragmentation, $F$ ) is affected by the choice of matrix. $F$ increases from $0.14 \pm 0.02$ for $m$-nitrobenzyl alcohol to $0.26 \pm 0.03$ for thioglycerol and to $0.28 \pm 0.02$ for glycerol under standard FAB conditions $(1 \mathrm{~mA}$ and $8 \mathrm{keV})$. This thermal effect has been noted elsewhere [18] and should not be limited to chemically labile analytes like those studied here. The mechanism of damage mitigation by thioglycerol and $m$-nitrobenzyl alcohol is the subject of ongoing studies.

\section{Parameter Interaction}

Compared with results for glycerol, the dependence of reduction on emission current (Figure 10a) or primary atom energy (Figure 10b) is greatly diminished when thioglycerol or m-nitrobenzyl alcohol is used as the matrix; fragmentation is affected similarly. In fact, measured intensities approach expected isotopic abundances in $m$-nitrobenzyl alcohol at virtually all emission currents and primary atom energies tested. Thus, the importance of the parameters in controlling chemical beam-induced damage for these systems can be ranked choice of matrix $>$ primary atom flux $>$ primary atom energy.

\section{Correlation with $E^{0}$}

Previous studies [5] sought to correlate the extent of beam-induced reduction of these dyes in FAB with published aqueous standard reduction potentials $\left(E^{0}\right)$. Assuming that (1) trends in reduction potentials are similar in water and glycerol, (2) intensities $(I)$ are directly proportional to concentrations, and (3) Nernstian behavior pertains in FAB, then $\ln \left[I_{\text {red }} / I_{\text {ox }}\right]$ should vary linearly with $E^{0}$. Figure 11 shows that this is not the 


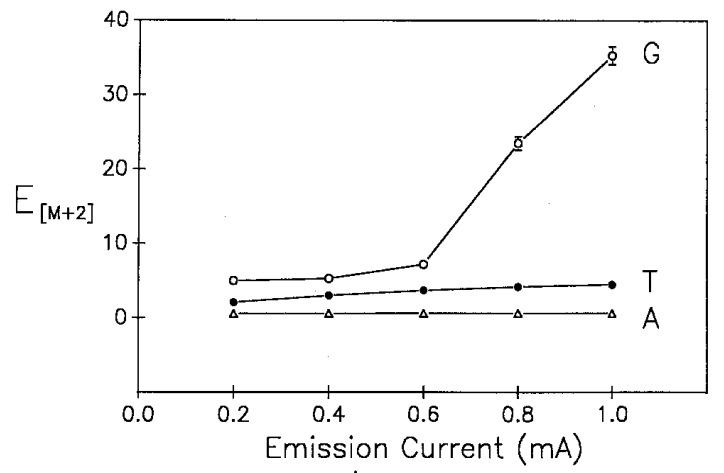

A

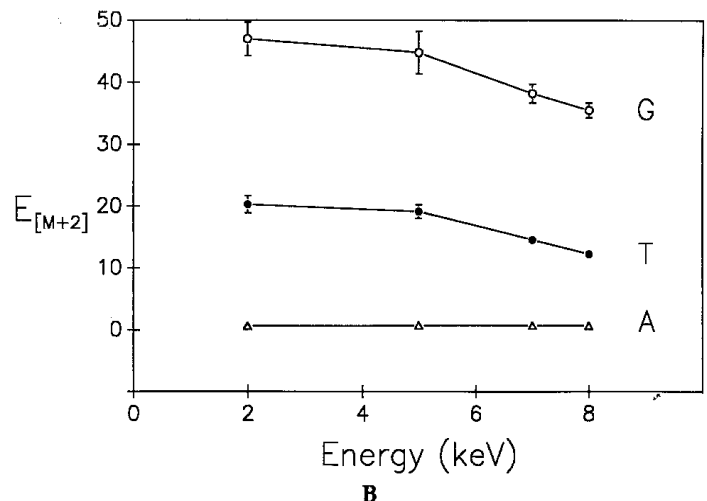

Figure 10. Extent of reduction $\left(E_{[\mathrm{M}+2]^{+}}\right)$of methylene blue versus (a) FAB gun emission current using 8-keV xenon atoms and (b) primary beam energy at an emission current of $0.2 \mathrm{~mA}$ for glycerol (G), thioglycerol (T), and m-nitrobenzyl alcohol (A).

case for $\ln \left(I_{[\mathrm{M}+2]^{+}} / I_{\mathrm{M}^{+}}\right)$for the series of dyes of Table 1 in the three solvents tested; similar plots were obtained for $\ln \left(I_{[\mathrm{M}+1]^{+}} / I_{\mathrm{M}^{+}}\right)$. Of the three underlying assumptions, the third is most likely to fail. The nonlinearity (perhaps not surprisingly) suggests, therefore, that both: kinetic and thermodynamic factors are important in controlling the extent of reduction in FAB.

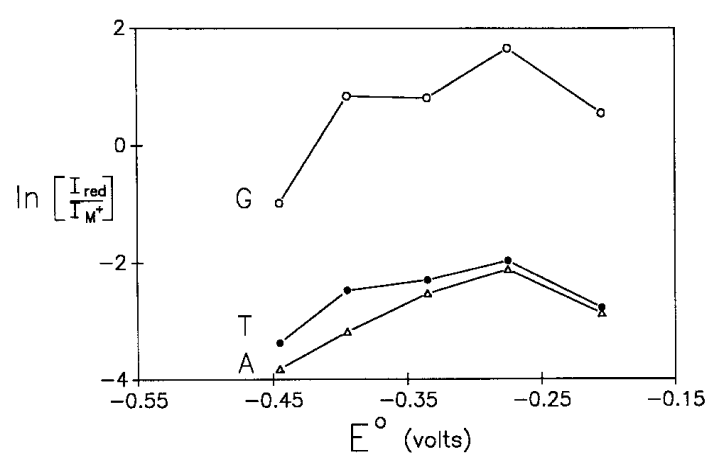

Figure 11. Intensity ratio $\ln \left[I_{\text {red }} / I_{\mathrm{M}^{+}}\right]$versus standard reduction potentials $\left(E^{0}\right)$ under standard $F A B$ conditions $(8 \mathrm{keV}$ and 1.0 $\mathrm{mA})$ for a series of dyes in glycerol $(\mathrm{G})$, thioglycerol $(\mathrm{T})$, and $m$-nitrobenzyl alcohol (A). $I_{\text {red }}$ is the intensity of the $[\mathrm{M}+2]^{+}$ ion corrected for isotopic contributions. See Table 1 for identity of dyes with indicated $E^{0}$ values.

\section{Conclusions}

It is clear from these results that the pervasive importance of FAB matrix selection extends even to the control of chemical and thermal beam-induced damage. More surprising is the fact that, for some systems, lower primary atom fluxes may actually improve analytical limits of detection while reducing beam-induced damage. Such knowledge of the relative sensitivity of $S / N$ and $S / B$ ratios and damage to changes in primary atom flux and energy can provide insight for future instrumental options; significant advantages of liquid SIMS over FAB in some cases may arise from the lower and better characterized fluxes accessible with ion (as opposed to atom) guns.

For samples susceptible to chemical damage, the use of lower fluxes may be especially important, reducing damage and improving $S / B, S / M_{x}$, and sample lifetimes. Even for samples not susceptible to chemical damage, use of lower fluxes can yield better $S / B$ values and sample lifetimes. However, for samples in which ionization is a result of beam-induced damage (i.e., analytes that do not yield preformed ions in solution), optimum conditions will depend upon the balance between beam-induced damage needed for ionization and $S / B$. This is the subject of ongoing studies, as are the mechanistic details behind these complex systems.

\section{Acknowledgments}

This work was supported in part by grants from the National Science Foundation (no. DMR-8406825, cofunded by the Army Research Office; and no. CHE-8822787). The UTK Chemistry Mass Spectrometry Center is funded by the Science Alliance, a State of Tennessee Center of Excellence. The NSF Chemical Instrumentation Program also contributed to the acquisition of the ZABEQ (no. CHE-8609251). Helpful discussions with Dr. Peter Todd (Oak Ridge National Laboratory) and Dr. Albert Tuinman (University of Tennessee) are gratefully acknowledged.

\section{References}

1. Barber, M.; Bordoli, R. S.; Sedgwick, R. D.; Tyler, A. N. J. Chem. Soc. Chem. Commun. 1981, 325.

2. Benninghoven, A.; Jaspers, D.; Sichterman, W. Appl. Phys. 1976, 11, 35.

3. Keough, T. Int. J. Mass Spectrom. Ion Processes 1988, 86, 155.

4. McCloskey, J. A.; Sethi, S. A.; Nelson, C. C. Anal. Chem. 1984, 56, 1975.

5. Pelzer, G.; De Pauw, E.; Dung, D. V.; Marien, J. J. Phys. Chem. 1984, 88, 5065.

6. Cerny, R. L.; Gross, M. L. Anal. Chem. 1985, 57, 1160.

7. Santana-Marques, M. G. O.; Ferrer-Correia, A. J. V.; Gross, M. L. Anal. Chem. 1989, 61, 1442.

8. Ashton, P. R.; Rose, M. E. Org. Mass Spectrom. 1986, 21, 388.

9. Brown, S. M.; Busch, K. L. Rapid Commun. Mass Spectrom. 1988, 2, 256.

10. Castro, M. E.; Mallis, L. M.; Russell, D. H. J. Am. Chem. Soc. 1985, 107, 5652.

11. Todd, P. J. Org. Mass Spectrom. 1988, 23, 419.

12. Aberth, W. H.; Burlingame, A. L. Anal. Chem. 1988, 60, 1426. 
13. Winger, B. E.; Hand, O. W.; Cooks, R. G. Int. J. Mass Spectrom. Ion Processes 1988, 84, 89.

14. Miller, J. M.; Balasanmugam, K.; Nye, J.; Deacon, G. B.; Thomas, N. C. Inorg. Chem. 1987, 26, 560.

15. Kyranos, J. N.; duSorbier, B. M.; Wronka, J.; Vouros, P.; Kirby, D. P. Org. Mass Spectrom. 1988, 23, 443.

16. Musselman, B.; Watson, J. T. Biomed. Environ. Mass Spectrom. 1987, 14, 247.

17. Callahan, J. H.; Hool, K.; Reynolds, J. D.; Cook, K. D. Anal. Chem. 1988, 60, 714 .

18. Takayama, M.; Fukai, T.; Nomura, T. Int. J. Mass Spectrom. Ion Processes 1989, 89, R1.

19. Alexander, A. J.; Hogg, A. M. Int. J. Mass Spectrom. Ion Processes 1986, 69, 297.

20. Ligon, W. V., Jr.; Dorn, S. B. Int. J. Mass Spectrom. Ion Processes 1986, 72, 317.

21. Gale, P. J.; Bentz, B. L.; Chait, B. T.; Field, F. H.; Cotter, R. J. Anal. Chem. 1986, 58, 1070.

22. McEwen, C. N.; Layton, S. F.; Taylor, S. K. Anal. Chem. $1977,49,922$.

23. Scheifers, S. M.; Verma, S.; Cooks, R. G. Anal. Chem. 1983, 55,2260 .
24. Pannell, L. K.; Sokoloski, E. A.; Fales, H. M.; Tate, R. L. Anal. Chem. 1985, 57, 1060.

25. Burinsky, D. J.; Dilliplane, R. L.; DiDonato, G. C.; Busch, K. L. Org. Mass Spectrom. 1988, 23, 231.

26. Ligon, W. V., Jr.; Dorn, S. B. Int. J. Mass Spectrom. Ion Processes 1987, 78, 99.

27. Bentz, B. L.; Gale, P. J. Int. J. Mass Spectrom. Ion Processes $1987,78,115$.

28. Mathias, A.; Williams, A. E.; Games, D. E.; Jackson, A. H. Org. Mass Spectrom. 1976, 11, 266.

29. Cook, K. D. Mass Spectrom. Rev. 1986, 5, 467.

30. Young, H. D. Statistical Treatment of Experimental Data; McGraw-Hill: New York, 1962; p 96.

31. Stimpson, B. P.; Evans, C. A., Jr. J. Electrostat. 1978, 5, 411.

32. Todd, P. J.; Groenewold, G. S. Anal. Chem. 1986, 58, 895.

33. Briggs, D.; Hearns, M. J. Int. J. Mass Spectrom. Ion Processes 1985, 67, 47.

34. Carter, G.; Colligan, J. S. Ion Bombardment of Solids; Elsevier: New York, 1968.

35. P. Todd, Oak Ridge National Laboratory; personal communication, 1989. 